\title{
Preterm-Term Birth Classification Using EMD-Based Time-Domain Features of Single-Channel Electrohysterogram Data
}

Suparerk Janjarasjitt ( $\square$ suparerk.j@ubu.ac.th )

Ubon Ratchathani University https://orcid.org/0000-0002-0252-8795

\section{Research Article}

Keywords: time-domain features, empirical mode decomposition, support vector machine, electrohysterogram, preterm birth

Posted Date: June 8th, 2021

DOI: https://doi.org/10.21203/rs.3.rs-570938/v1

License: (c) (1) This work is licensed under a Creative Commons Attribution 4.0 International License. Read Full License

Version of Record: A version of this preprint was published at Physical and Engineering Sciences in Medicine on August 31st, 2021. See the published version at https://doi.org/10.1007/s13246-021-01051W. 


\title{
Preterm-Term Birth Classification Using EMD-Based Time-Domain Features of Single-Channel \\ Electrohysterogram Data
}

\section{Suparerk Janjarasjitt}

\begin{abstract}
The preterm birth anticipation is a crucial task that can reduce the rate of preterm birth and also the complications of preterm birth. Electrohysterogram (EHG) or uterine electromyogram (EMG) data have been evidenced that they can provide an information useful for preterm birth anticipation. Four distinct time-domain features, i.e., mean absolute value, average amplitude change, difference absolute standard deviation value, and log detector, commonly applied to EMG signal processing are applied and investigated in this study. A single-channel of EHG data is decomposed into its constituent components, i.e., intrinsic mode functions, using empirical mode decomposition (EMD) before their time-domain features are extracted. The time-domain features of intrinsic mode functions of EHG data associated with preterm and term births are applied for preterm-term
\end{abstract}

S. Janjarasjitt

Department of Electrical and Electronic Engineering, Ubon Ratchathani University, 85 Sathonlamak Road, Warin Chamrap, Ubon Ratchathani, Thailand 34190

Tel.: +66-4535-3332

Fax: +66-4535-3333

E-mail: suparerk.j@ubu.ac.th 
birth classification using support vector machine (SVM) with a radial basis function. The preterm-term classifications are validated using 10 -fold cross validation. From the computational results, it is shown that the excellent preterm-term birth classification can be achieved using a single-channel of EHG data. The computational results further suggest that the best overall performance on preterm-term birth classification is obtained when thirteen (out of sixteen) EMD-based timedomain features are applied. The best accuracy, sensitivity, specificity, and $F_{1^{-}}$ score achieved are, respectively, 0.9382, 0.9130, 0.9634, and 0.9366.

Keywords time-domain features · empirical mode decomposition · support vector machine $\cdot$ electrohysterogram $\cdot$ preterm birth

\section{Introduction}

Preterm birth or premature birth is defined by the World Health Organization (WHO) [1] as all births before 37 completed weeks of gestation [2]. Preterm birth is one of major health concerns as a preterm birth gives the baby less time to develop in the womb [3] and also a major cause of death [2]. Preterm birth can cause short-term complications and also long-term complications [3]. Solutions for reducing preterm birth include improving health care before, between and during pregnancies, providing effective treatments and interventions, and identifying women at risk for preterm delivery $[4,5]$. A preterm birth anticipation is therefore one of crucial tasks that useful in reducing preterm birth and its complications.

Electrohysterogram (EHG) or uterine electromyogram (EMG) measuring the electrical activity of uterine muscles [6] becomes a potential noninvasive tool for assessing and monitoring uterine activity [6-8]. There have been a number of com- 
putational tools and techniques applied for classifying and predicting preterm birth as some of those summarized in Table 1. From the previous studies, the root mean square, the peak frequency, the median frequency, and the sample entropy are the most common quantitative features of EHG signals extracted and applied $[6,9,10]$. Time-domain features of EHG signals as well as the root mean square [11] are also investigated. Furthermore, Hemthanon et al. [12] examine the correlation between the time-domain features of EHG signals and gestational age. A pre-processing stage such as wavelet decomposition [10] and empirical mode decomposition [13], may be applied to EHG signals before quantitative features are extracted in a number of studies. Various classifiers including random forest $[10,13]$, support vector machine (SVM) [13], and artificial neural network [13], are applied. In addition to machine learning techniques, basic statistical analyses such as $t$-test [6] and Wilcoxon rank sum test [14] are applied to validate the performance on preterm and term birth discrimination. Improved classification and discrimination results are obtained when oversampling techniques are also applied to enable a balanced number of preterm and term birth $[10,13,9]$.

In this study, the capability of time-domain features of a single-channel EHG data on preterm-term birth classification is further examined as time-domain features have been common and useful quantitative features applied for various applications of EMG signal processing and analysis. Four time-domain features including the mean absolute value, the average amplitude change, the difference absolute standard deviation value, and the log detector are determined. Empirical mode decomposition (EMD) is applied to decompose a single-channel EHG data into a finite set of oscillatory components, called intrinsic mode functions (IMFs), associating with sequentially decreasing frequencies [15-17]. 
2 Methodology

2.1 Dataset

The electrohysterogram (EHG) dataset examined in this study is obtained from EHG recordings collected at the Department of Obstetrics and Gynecology, Medical Centre Ljubljana Ljubljana [6,18] from 1997 until 2006. Three hundred EHG recordings are publicly available at https ://www.physionet.org/content/tpehgdb/ on the PhysioNet [18]. The EHG recordings were recorded from a general population of pregnant women during regular check-ups either around the 22nd week of gestation or around the 32nd week of gestation $[6,18]$. Three channels of EHG recordings obtained from four electrodes placed around the navel $[6,18]$ are referred to as $s_{1}, s_{2}$, and $s_{3}$. The EHG data were acquired using the sampling frequency of $20 \mathrm{~Hz}$.

The EHG dataset is classified into four classes, referred to as PE, PL, TE, and TL, corresponding to two subject groups and two periods of recordings summarized in Table 2. Those two subject groups are a group of preterm birth $(\mathrm{P})$ which is associated with the delivery before or on the 37th week of gestation and a group of term birth $(\mathrm{T})$ which is associated with the delivery was after the 37th of gestation $[6,18]$. Those two periods of recordings are an early period (E) which is associated with the recordings acquired before the 26th week of gestation and a late period (L) which is associated with the recordings acquired during or after the 26th week of gestation [6,18]. There are 19, 19, 143, and 119 of EHG recordings associated with the PE, PL, TE, and TL classes, respectively. 
2.2 Data Processing

EHG signals corresponding to each channel of original EHG recordings are segmented with a length of 14400 samples, equivalently 720 seconds, from the end of EHG recordings. The EHG segments are further divided into 3600-sample epochs with 50\% overlap. Accordingly, there are twelve EHG epochs for each EHG segment. EHG epochs are dissected and decomposed into four intrinsic mode functions (IMFs) using the empirical mode decomposition (EMD) $[15,17,19]$. Time-domain features which are commonly applied in EMG signal processing and analysis are subsequently extracted from the intrinsic mode functions of EHG epochs. The mean absolute value (MAV), the average amplitude change (AAC), the difference absolute standard deviation value (DASDV), and the log detector are the four distinct time-domain features applied for preterm-term birth classification.

Denote a sequence of intrinsic mode functions $x[n]$ with a length of $N$ applied for time-domain feature extraction. The time-domain features of each EHG epoch, respectively referred to as $F_{1}, F_{2}, F_{3}$, and $F_{4}$, are given as follows

- Mean absolute value (MAV):

$$
F_{1}=\frac{1}{N} \sum_{n=0}^{N-1}|x[n]| .
$$

- Average amplitude change (AAC):

$$
F_{2}=\frac{1}{N} \sum_{n=0}^{N-1}|x[n+1]-x[n]| .
$$

- Difference absolute standard deviation value (DASDV):

$$
F_{3}=\sqrt{\frac{1}{N-1} \sum_{n=0}^{N-1}(x[n+1]-x[n])^{2}} .
$$

- Log detector:

$$
F_{4}=e^{\frac{1}{N} \sum_{n=0}^{N-1} \log (|x[n]|)} .
$$


2.3 Classification and Evaluation

A feature vector applied for preterm-term birth classification is composed of timedomain features of intrinsic mode functions of EHG epochs. A number of timedomain features used to form the feature vector ranges from 2 to 16, i.e., all of the time-domain features. The time-domain features of intrinsic mode functions of EHG epochs selected and applied correspond to their ranking scores obtained from the $p$-value of $\chi^{2}$ test, i.e., $\log (p)$. There are two preterm-term birth classifications examined in this study: 1) PE-TE classification and 2) PL-TL classification. Support vector machines (SVMs) are applied for the preterm-term birth classifications. In the training of SVM models, the kernel function used is the radial basis function $(\mathrm{RBF})$ with the standardization applied.

For the evaluation of preterm-term birth classifications, the 10-fold crossvalidation is applied. The performance of preterm birth classifications is evaluated using four conventional classification performance measures including accuracy (Ac), sensitivity (Se), specificity (Sp), precision, recall, and $F_{1}$-score, which are, respectively, given by

$$
\begin{aligned}
\mathrm{Ac} & =\frac{\mathrm{TP}+\mathrm{TN}}{\mathrm{TP}+\mathrm{TN}+\mathrm{FP}+\mathrm{FN}}, \\
\mathrm{Se} & =\frac{\mathrm{TP}}{\mathrm{TP}+\mathrm{FN}}, \\
\mathrm{Sp} & =\frac{\mathrm{TN}}{\mathrm{TN}+\mathrm{FP}}, \\
F_{1}-\text { score } & =\frac{\mathrm{TP}}{\mathrm{TP}+\frac{1}{2}(\mathrm{FP}+\mathrm{FN})}
\end{aligned}
$$

where TP, TN, FP, and FN denote a number of true positives, a number of true negatives, a number of false positives, and a number of false negatives, respectively. 


\section{Results}

3.1 Preterm-Term Birth Classifications Using Early Period EHG Data

The accuracy, sensitivity, specificity, and $F_{1}$-score of the preterm-term birth classification obtained using the channels, $s_{1}, s_{2}$, and $s_{3}$, of early period EHG data are shown in Fig. 1(a)-(d), Fig. 2(a)-(d), and Fig. 3(a)-(d), respectively. The accuracy, sensitivity, specificity, and $F_{1}$-score of the preterm-birth classification range, respectively, from 0.7002 to $0.9382,0.5515$ to $0.9153,0.6774$ to 0.9634 , and 0.6479 to 0.93662 . The performance of the preterm-term birth classification corresponding to all metrics tend to be boosted as the number of time-domain features of intrinsic mode functions of EHG epochs applied increases.

The best performances obtained from the preterm-term birth classifications using the time-domain features of intrinsic mode functions of early period EHG data corresponding to each performance metrics are summarized in Table 3. All of performance metrics, i.e., the accuracy, sensitivity, specificity, and $F_{1}$-score, of the preterm-term birth classification obtained using the channel $s_{1}$ of early period EHG data are completely higher than those of the preterm-term birth classification using either channel $s_{2}$ or $s_{3}$. The best performance of the preterm-term birth classifications is generally achieved using thirteen time-domain features of intrinsic mode functions of channel $s_{1}$ of early period EHG data in which the best accuracy, specificity, and $F_{1}$-score are obtained. The accuracy, sensitivity, specificity, and $F_{1-}$ score of the preterm-term birth classification using thirteen time-domain features of intrinsic mode functions of channel $s_{1}$ of early period EHG data are 0.9382, 0.9130, 0.9634, and 0.9366, respectively. 
3.2 Preterm-Term Birth Classifications Using Late Period EHG Data

The accuracy, sensitivity, specificity, and $F_{1}$-score of the preterm-term birth classification obtained using the channels, $s_{1}, s_{2}$, and $s_{3}$, of late period EHG data are, respectively, shown in Fig. 4(a)-(d), Fig. 5(a)-(d), and Fig. 6(a)-(d). The accuracy, sensitivity, specificity, and $F_{1}$-score of the preterm-birth classification range, respectively, from 0.6842 to $0.8970,0.5858$ to $0.9176,0.6728$ to 0.8993 , and 0.6498 to 0.8980 . The trend of increasing accuracy, sensitivity, specificity, and $F_{1}$-score of the preterm-term birth classifications as the number of time-domain features of intrinsic mode functions of EHG epochs applied increases is obviously evidenced in the channels $s_{1}$ and $s_{3}$.

Table 4 summarizes the best performances obtained from the preterm-term birth classifications using the time-domain features of intrinsic mode functions of late period of EHG data corresponding to each performance metrics. Likewise, the higher values of accuracy, sensitivity, specificity, and $F_{1}$-score, of the pretermterm birth classifications using late period EHG data are obtained in the channel $s_{1}$ compared to the channels $s_{2}$ and $s_{3}$. The best accuracy and $F_{1}$-score of the preterm-term birth classifications are obtained using thirteen time-domain features of intrinsic mode functions of channel $s_{1}$ of late period EHG data. The accuracy, sensitivity, specificity, and $F_{1}$-score of the preterm-term birth classification using thirteen time-domain features of intrinsic mode functions of channel $s_{1}$ of late period EHG data are, respectively, 0.8970, 0.9062, 0.8879, and 0.8980 . 
4 Discussion

From the computational results, it is shown that the performance on preterm-term birth classification using time-domain features is significantly improved when the empirical mode decomposition (EMD) is applied to decompose a single-channel of EHG data into intrinsic mode functions. Only four time-domain features, i.e., the mean absolute value, the average amplitude change, the difference absolute standard deviation value, and the log detector, are applied compared to seventeen time-domain features of original (full-bandwidth) and filtered EHG data examined in the previous study [11]. The product of sensitivity and specificity of pretermterm birth classification is increased from 0.5134 to 0.8796 .

The better performance on preterm-term birth classification is achieved using the channel $s_{1}$ of EHG data which records the difference of electrical activity of uterine muscles above the navel [6]. The number of time-domain features of intrinsic mode functions of single-channel EHG data providing the best performance on preterm-term birth classifications is 13 . The accuracy, sensitivity, specificity, and $F_{1}$-score of preterm-term birth classification using thirteen time-domain features of intrinsic mode functions of single-channel EHG data acquired in the early period are, respectively, 0.9382, 0.9130, 0.9634, and 0.9366 while the accuracy, sensitivity, specificity, and $F_{1}$-score of preterm-term birth classification using thirteen timedomain features of intrinsic mode functions of single-channel EHG data acquired in the late period are $0.8970,0.9062,0.8879$, and 0.8980 , respectively. Interestingly, the better performance can be obtained from the preterm-term birth classification using single-channel EHG data acquired in the early period, i.e., before the 26th 
week of gestation compared those acquired in the late period, i.e., during or after the 26 th week of gestation.

The performance on preterm-term birth classification and discrimination of notable previous studies summarized in Table 1 are compared in Table 5. Regarding the previous studies $[9,10]$ examining and reporting comparable performance metrics, the performance on preterm-term birth classification obtained using the time-domain features of intrinsic mode functions of single-channel of EHG data is shown to be generally superior to the best accuracy, sensitivity, and specificity of 0.92 [10], 0.92 [9], and 0.96 [10]. All four time-domain features applied in this study therefore reveal distinguishing characteristics of EHG data which are useful for preterm-term birth classification.

\section{Conclusion}

Four distinct time-domain features, i.e., the mean absolute value, the average amplitude change, the difference absolute standard deviation value, and the log detector, commonly applied for EMG signal processing and analysis are extracted from the first four intrinsic mode functions of single-channel of EHG data. The computational results suggest that the number of time-domain features of intrinsic mode functions of EHG data required for preterm-term birth classification is 13 (out of 16 ). The accuracy, sensitivity, specificity, and $F_{1}$-score of preterm-term birth classification using thirteen time-domain features of intrinsic mode functions of channel $s_{1}$ of EHG data acquired in the early period are $0.9382,0.9130,0.9634$, and 0.9366 , respectively. 


\section{Compliance with Ethical Standards}

Funding: This study was funded by a TRF Research Career Development Grant, jointly funded by Thailand Research Fund (TRF) and Ubon Ratchathani University (contract number RSA6180041).

Conflict of Interest: The author declares that he has no conflict of interest.

\section{References}

1. WHO. WHO: Recommended definitions, terminology and format for statistical tables related to the perinatal period and use of a new certificate for cause of perinatal deaths, volume $=56$, pages $=247-253$, year $=1976$, journal $=$ Acta Obstetricia et Gynecologica Scandinavica;

2. Blencowe H, Cousens S, Chou D, Oestergaard M, Say L, Moller AB, et al. Born Too Soon: The global epidemiology of 15 million preterm births. Reproductive Health. 2013;10(suppl 1):S2.

3. Mayo Clinic. Premature birth; 2021 [cited May 27, 2021]. Available from: https: //www.mayoclinic.org/diseases-conditions/premature-birth/symptoms-causes/ syc-20376730.

4. Wolrd Health Organization. Preterm birth; 2018 [cited May 27, 2021]. Available from: https://www.who.int/news-room/fact-sheets/detail/preterm-birth.

5. Centers for Disease Control and Prevention. Preterm birth; 2020 [cited May 27, 2021]. Available from: https://www.cdc.gov/reproductivehealth/maternalinfanthealth/ pretermbirth.htm.

6. Fele-Žorž G, Kavšek G, Novak-Antolič v, Jager F. A comparison of various linear and nonlinear signal processing techniques to separate uterine EMG records of term and pre-term delivery groups. Med Biol Eng Comput. 2008;46:911-922.

7. Maner WL, Garfield RE. Identification of human term and preterm labor using artificial neural networks on uterine electromyography data. Ann Biomed Eng. 2007;35:465-473. 
8. Leman H, Marque C, Gondry J. Use of the electrohysterogram signal for characterization of contractions during pregnancy. IEEE Trans Biomed Eng. 1999;46:1222-1229.

9. Fergus P, Cheung P, Hussain A, Al-Jumeily D, Dobbins C, Iram S. Prediction of preterm deliveries from EHG signals using machine learning. PLoS ONE. 2013;8:e77154.

10. Peng J, Hao D, Yang L, Du M, Song X, Jiang H, et al. Evaluation of electrohysterogram measured from different gestational weeks for recognizing preterm delivery: a preliminary study using random forest. Biocybernetics and Biomedical Engineering. 2020;40:352-362.

11. Hemthanon C, Janjarasjitt S. Examination of time-domain features of EHG data for preterm-term birth classification. Journal of Computers. 2019;30:41-54.

12. Hemthanon C, Janjarasjitt S. Correlation Between Time-Domain Features of Electrohysterogram Data of Pregnant Women and Gestational Age. In: Lin KP, Magjarevic R, de Carvalho P, editors. IFMBE Proceedings. vol. 74; 2020. p. 212-218.

13. Ren P, Yao S, Li J, Valdes-Sosa PA, Kendrick KM. Improved prediction of preterm delivery using empirical mode decomposition analysis of uterine electromyography signals. PLoS ONE. 2015;10:e0132116.

14. Mas-Cabo J, Ye-Lin Y, Garcia-Casado J, Díaz-Martinez A, Perales-Marin A, MonfortOrtiz R, et al. Robust characterization of the uterine myoelectrical activity in different obstetric scenarios. Entropy. 2020;22:743.

15. Huang NE, Shen Z, Long SR, Wu MC, Shih HH, Zheng Q, et al. The empirical mode decomposition and the Hilbert spectrum for nonlinear and non-stationary time series analysis. Proceedings of the Royal Society A. 1998;454:903-995.

16. Huang NE, Shen SS. Hilbert-Huang Transform and Its Applications. 2nd ed. New Jersey: World Scientific; 2014.

17. Fontugne R, Borgnat P, Flandrin P. Online empirical mode decomposition. In: 2017 IEEE International Conference on Acoustics, Speech and Signal Processing (ICASSP); 2017. p. 4306-4310.

18. Goldberger AL, Amaral LAN, Glass L, Hausdorff JM, Ivanov PC, Mark RG, et al. PhysioBank, PhysioToolkit, and PhysioNet: Components of a New Research Resource for Complex Physiologic Signals. Circulation. 2000;101(23):e215-e220.

19. Huang NE, Wu MLC, Long SR, Shen SSP, Qu W, Gloersen P, et al. A confidence limit for the empirical mode decomposition and Hilbert spectral analysis. Proceedings of the 
Royal Society A. 2003;459:2317-2345. 
Table 1 Previous studies on preterm birth classification and discrimination using EHG data

\begin{tabular}{|c|c|c|}
\hline Ref. & First author surname & Description \\
\hline$[6]$ & Fele-Žorž et al. & $\begin{array}{l}\text { Features: root mean square; peak frequency; median fre- } \\
\text { quency; autocorrelation zero-crossing; Lyapunov exponent; } \\
\text { correlation dimension; sample entropy } \\
\text { Statistical analysis: } t \text {-test }\end{array}$ \\
\hline$[9]$ & Fergus et al. & $\begin{array}{l}\text { Features: root mean square; peak frequency; median fre- } \\
\text { quency; sample entropy } \\
\text { Dimensionality reduction: principal component analysis } \\
\text { Classifier: linear discriminant classifier; quadratic discrimi- } \\
\text { nant classifier; uncorrelated normal density based classifier; } \\
\text { polynomial classifier; logistic classifier; 3-nearest neighbor; } \\
\text { decision tree; parzen classifier; support vector classifier } \\
\text { Oversampling technique: SMOTE }\end{array}$ \\
\hline [10] & Peng et al. & $\begin{array}{l}\text { Features: root mean square; autocorrelation zero-crossing; } \\
\text { peak frequency; median frequency; wavelet-based features; } \\
\text { time reversability; Lyapunov exponent; sample entropy; } \\
\text { correlation dimension } \\
\text { Classifier: random forest } \\
\text { Oversampling technique: adaptive synthetic sampling }\end{array}$ \\
\hline [13] & Ren et al. & $\begin{array}{l}\text { Pre-processing: empirical mode decomposition } \\
\text { Features: Shannon entropy } \\
\text { Classifier: support vector machine; random forest; artificial } \\
\text { neural network; AdaBoost; Bayesian network } \\
\text { Oversampling technique: SMOTE }\end{array}$ \\
\hline$[14]$ & Mas-Cabo et al. & $\begin{array}{l}\text { Features: root mean square; dominant frequency; decile of } \\
\text { power spectrum; spectral moment ratio } \\
\text { Statistical analysis: Wilcoxon rank sum test }\end{array}$ \\
\hline [11] & Hemthanon et al. & $\begin{array}{l}\text { Features: integrated EMG; mean absolute value; modified } \\
\text { mean absolute value; simple square integral; absolute value } \\
\text { of temporal moments; root mean square; } \nu \text {-order; log detec- } \\
\text { tor; waveform length; average amplitude change; difference } \\
\text { absolute standard deviation value; zero crossing; slope sign } \\
\text { change } \\
\text { Feature selection: Pearson's correlation coefficient; } t \text {-test } \\
\text { Classifier: support vector machine }\end{array}$ \\
\hline
\end{tabular}


Table 2 Classes of EHG Dataset

\begin{tabular}{clll}
\hline Class & Subject group & Period of recordings & Number of recordings \\
\hline PE & Preterm birth $(\mathrm{P})$ & Early period (E) & 19 \\
$\mathrm{PL}$ & Preterm birth $(\mathrm{P})$ & Late period (L) & 19 \\
$\mathrm{TE}$ & Term birth $(\mathrm{T})$ & Early period $(\mathrm{E})$ & 143 \\
$\mathrm{TL}$ & Term birth $(\mathrm{T})$ & Late period (L) & 119 \\
\hline
\end{tabular}


Table 3 Best Performance on Preterm-Term Birth Classification Using Early Period EHG Data

\begin{tabular}{llll}
\hline Channel & Metric & Best performance & No. of features \\
\hline$s_{1}$ & Ac & 0.9382 & 13 \\
& Se & 0.9153 & 9 \\
& Sp & 0.9634 & 13 \\
& $F_{1}$-Score & 0.9366 & 13 \\
\hline$s_{2}$ & Ac & 0.8936 & 16 \\
& Se & 0.8673 & 16 \\
& Sp & 0.9245 & 14 \\
& $F_{1}$-Score & 0.8907 & 16 \\
\hline$s_{3}$ & Ac & 0.8513 & 10 \\
& Se & 0.8101 & 10 \\
& Sp & 0.9199 & 10 \\
\hline & $F_{1}$-Score & 0.8449 & \\
\hline
\end{tabular}


Table 4 Best Performance on Preterm-Term Birth Classification Using Late Period EHG

Data

\begin{tabular}{|c|c|c|c|}
\hline Channel & Metric & Best performance & No. of features \\
\hline \multirow[t]{4}{*}{$s_{1}$} & $\mathrm{Ac}$ & 0.8970 & 13 \\
\hline & $\mathrm{Se}$ & 0.9176 & 16 \\
\hline & $\mathrm{Sp}$ & 0.8993 & 12 \\
\hline & $F_{1}$-Score & 0.8980 & 13 \\
\hline \multirow[t]{4}{*}{$s_{2}$} & $\mathrm{Ac}$ & 0.8387 & 9 \\
\hline & $\mathrm{Se}$ & 0.8673 & 15 \\
\hline & $\mathrm{Sp}$ & 0.8650 & 8 \\
\hline & $F_{1-\text { Score }}$ & 0.8366 & 15 \\
\hline \multirow[t]{4}{*}{$s_{3}$} & $\mathrm{Ac}$ & 0.8387 & 10 \\
\hline & $\mathrm{Se}$ & 0.8787 & 16 \\
\hline & $\mathrm{Sp}$ & 0.8192 & 10 \\
\hline & $F_{1}$-Score & 0.8418 & 10 \\
\hline
\end{tabular}


Table 5 Comparison of performance on preterm-term birth classification and discrimination

\begin{tabular}{lll}
\hline Ref. & First author surname & Best performance \\
\hline$[6]$ & Fele-Žorž et al. & $p$-value: 0.011 \\
\hline$[9]$ & Fergus et al. & Area under curve: 0.89 \\
& & Sensitivity: 0.92 \\
\hline$[10]$ & Peng et al. & Accuracy: 0.92 \\
& & Sensitivity: 0.88 \\
& & Specificity: 0.96 \\
& & Area under curve: 0.88 \\
\hline$[13]$ & Area under curve: 0.986 \\
\hline$[14]$ & Ren et al. & $p$-value: $<0.01$ \\
\hline Mas-Cabo et al. & Product of sensitivity and specificity: 0.5134 \\
\hline Current study & Janjarasjitt & Accuracy: 0.9382 \\
& & Sensitivity: 0.9130 \\
& & Specificity: 0.9634 \\
& & $F_{1}$-score: 0.9366 \\
& & Product of sensitivity and specificity: 0.8796 \\
& &
\end{tabular}




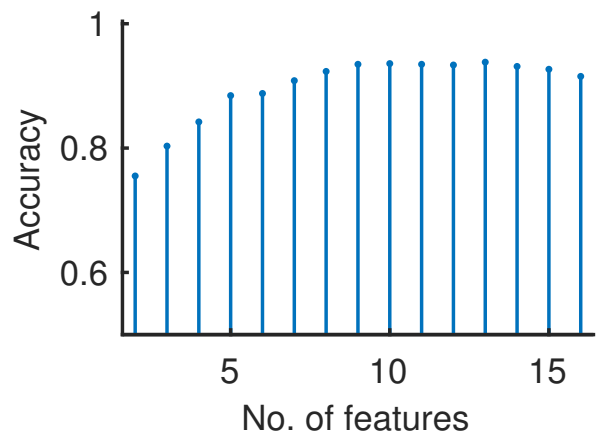

(a) Accuracy

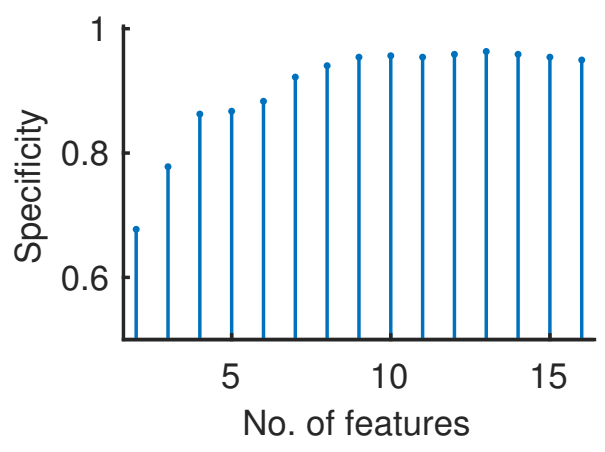

(c) Specificity

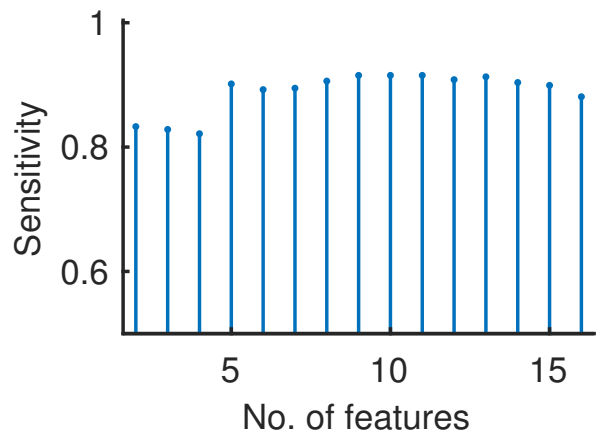

(b) Sensitivity

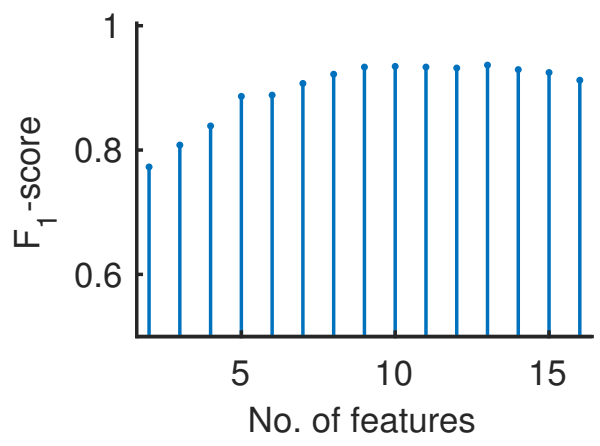

(d) $F_{1}$-score

Fig. 1 Performance of the preterm-term birth classification obtained using the channel $s_{1}$ of early period EHG data. 


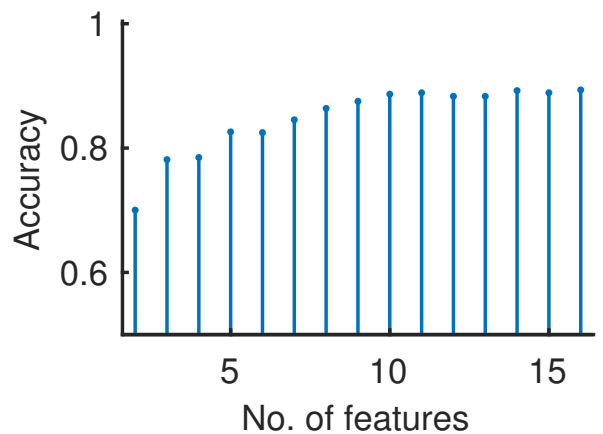

(a) Accuracy

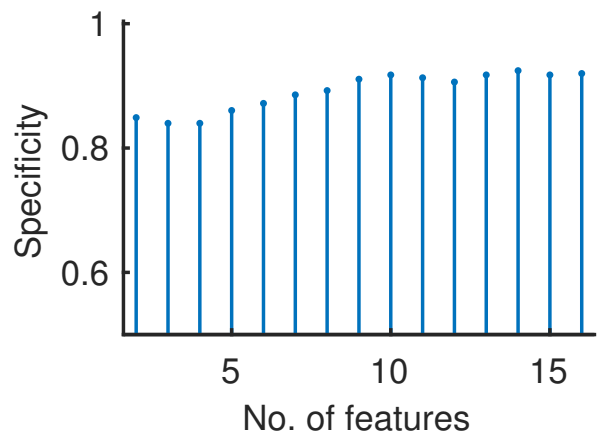

(c) Specificity

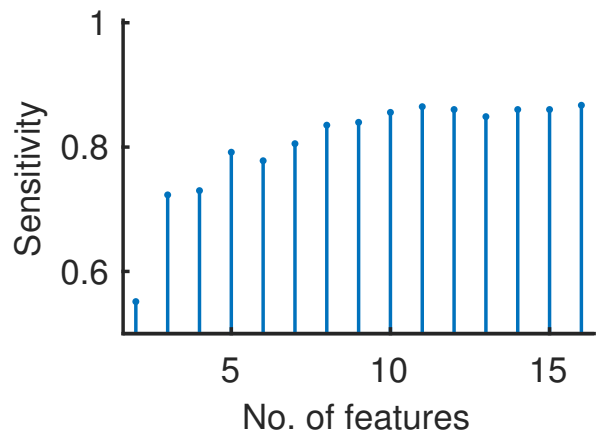

(b) Sensitivity

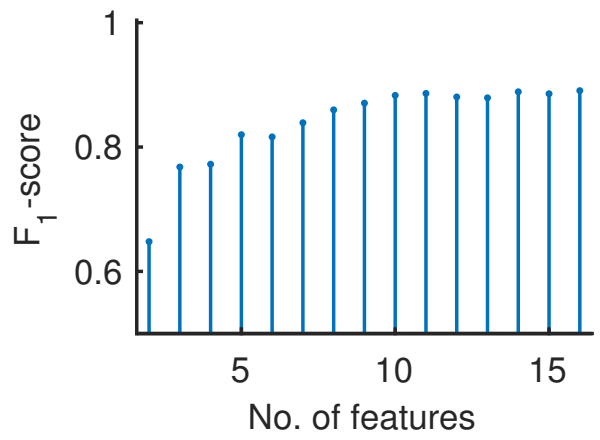

(d) $F_{1}$-score

Fig. 2 Performance of the preterm-term birth classification obtained using the channel $s_{2}$ of early period EHG data. 


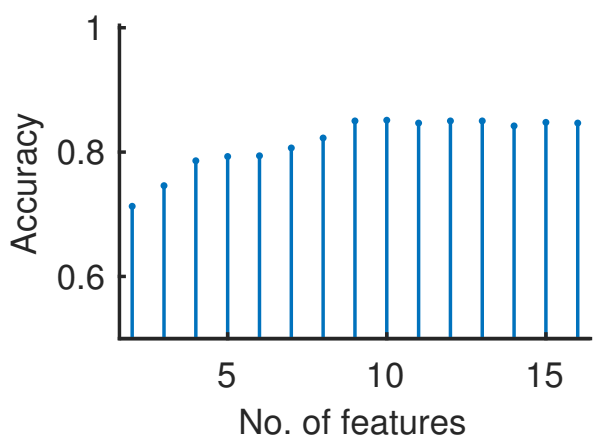

(a) Accuracy

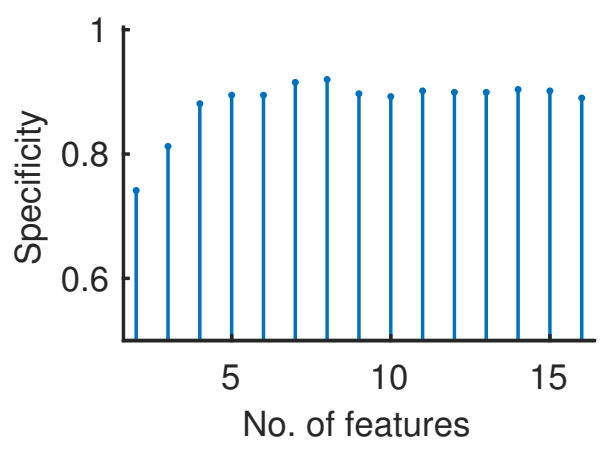

(c) Specificity

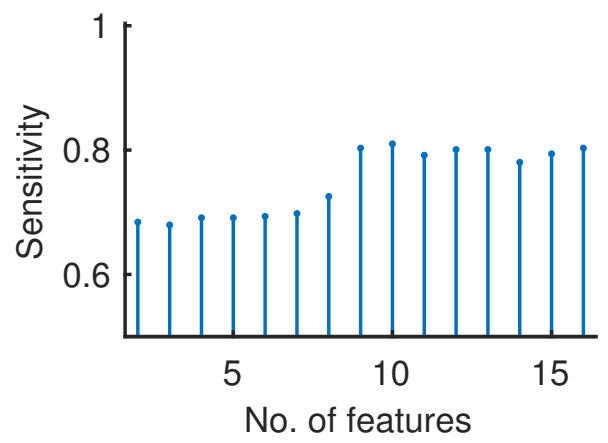

(b) Sensitivity

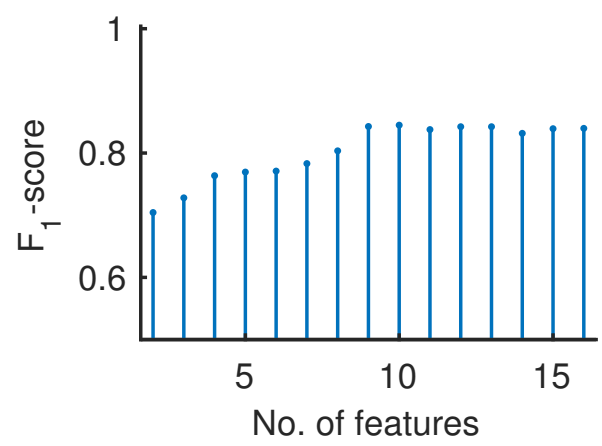

(d) $F_{1}$-score

Fig. 3 Performance of the preterm-term birth classification obtained using the channel $s_{3}$ of early period EHG data. 


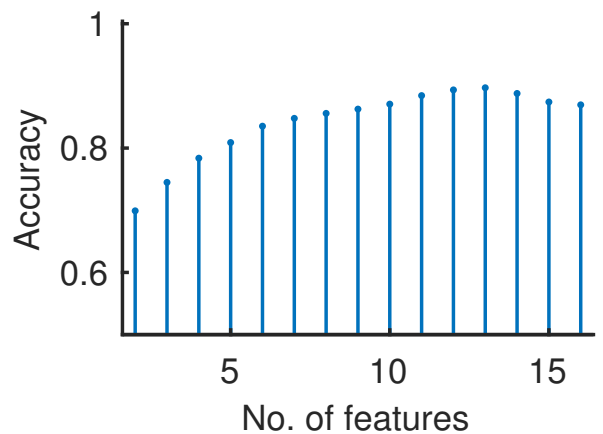

(a) Accuracy

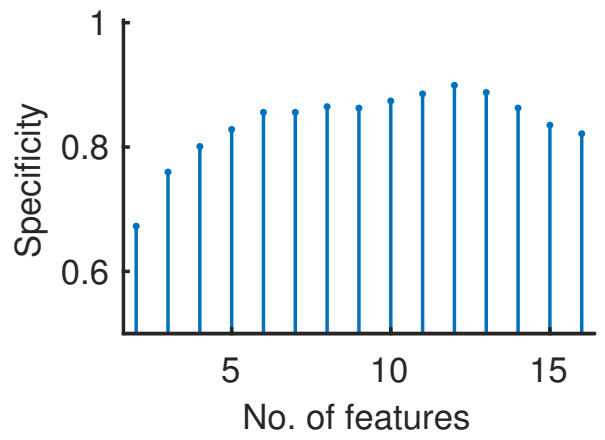

(c) Specificity

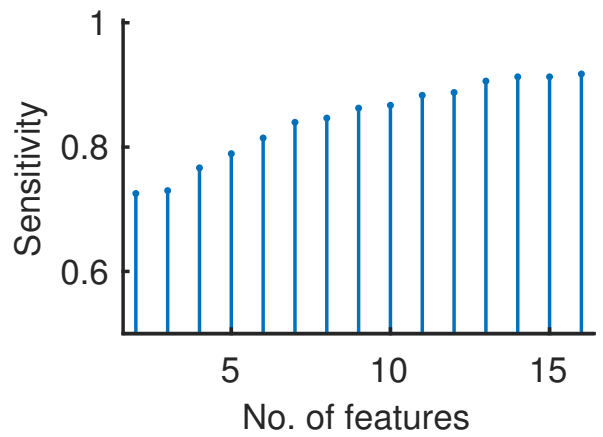

(b) Sensitivity

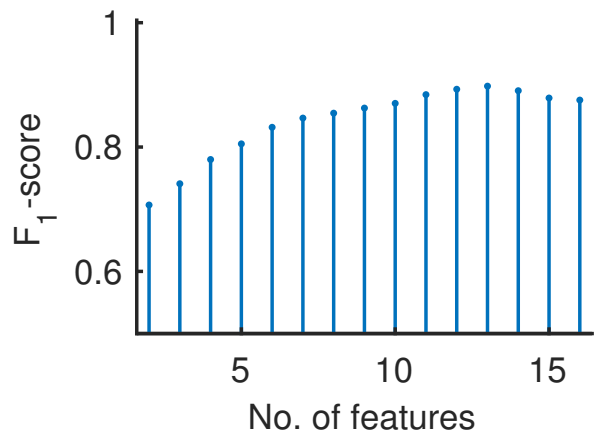

(d) $F_{1}$-score

Fig. 4 Performance of the preterm-term birth classification obtained using the channel $s_{1}$ of late period EHG data. 


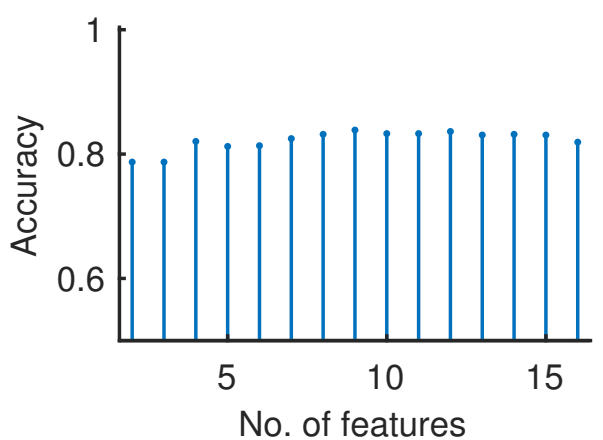

(a) Accuracy

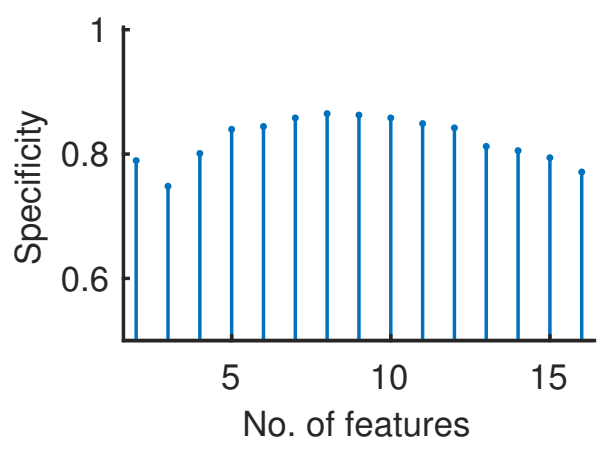

(c) Specificity

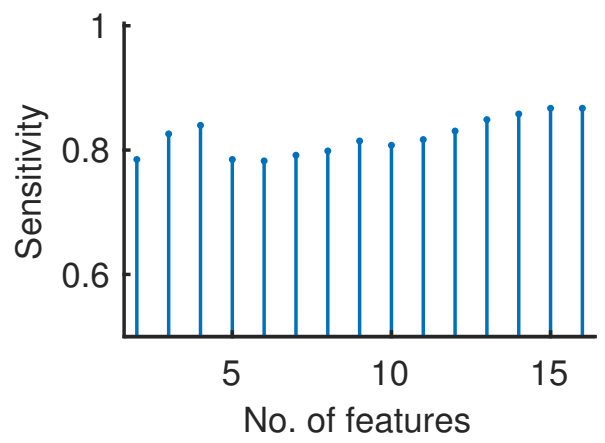

(b) Sensitivity

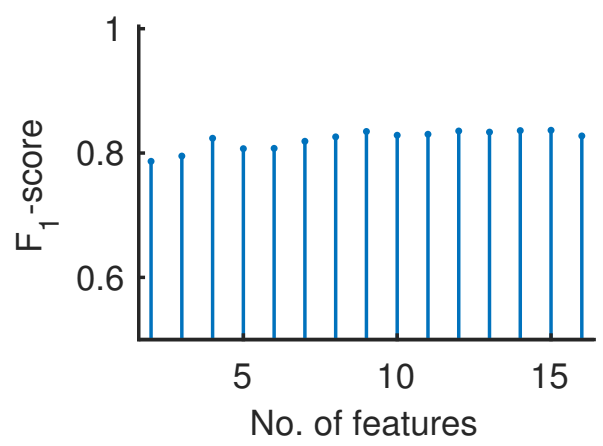

(d) $F_{1}$-score

Fig. 5 Performance of the preterm-term birth classification obtained using the channel $s_{2}$ of late period EHG data. 


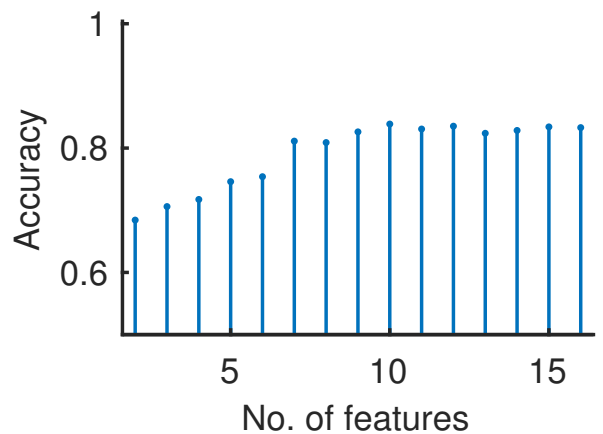

(a) Accuracy

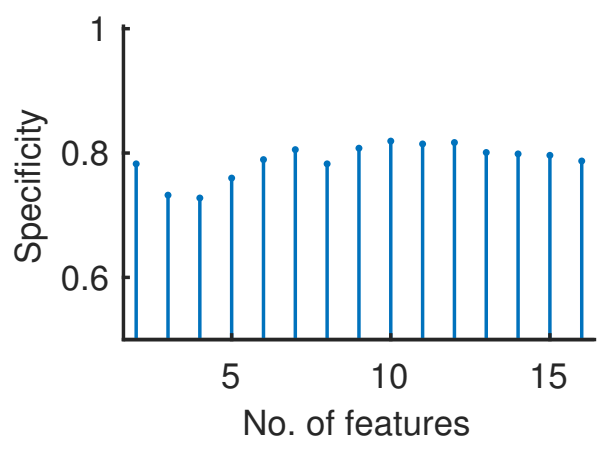

(c) Specificity

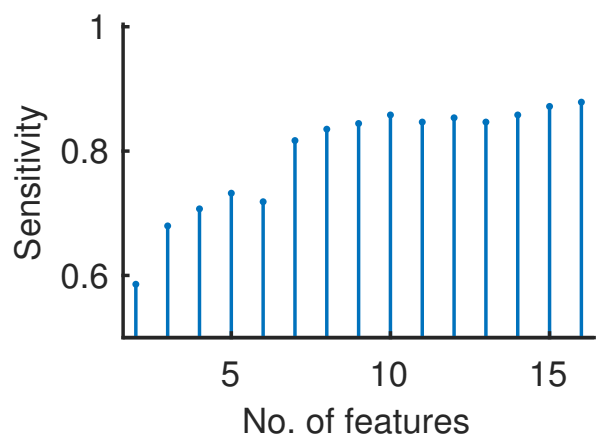

(b) Sensitivity

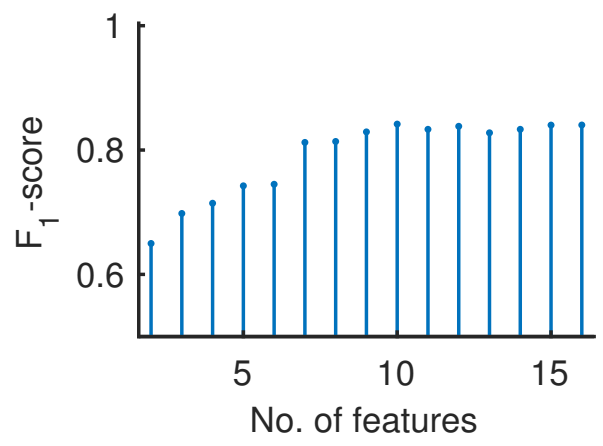

(d) $F_{1}$-score

Fig. 6 Performance of the preterm-term birth classification obtained using the channel $s_{3}$ of late period EHG data. 
Figures

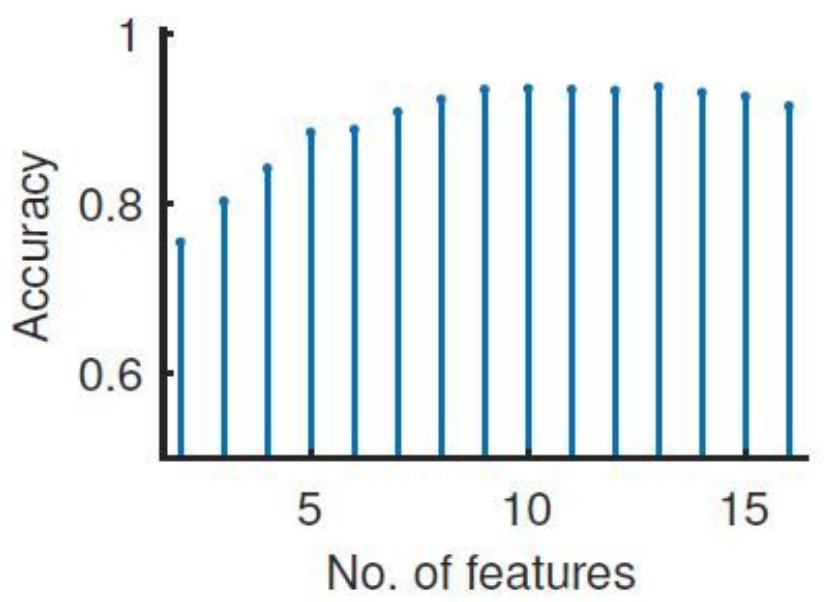

(a) Accuracy

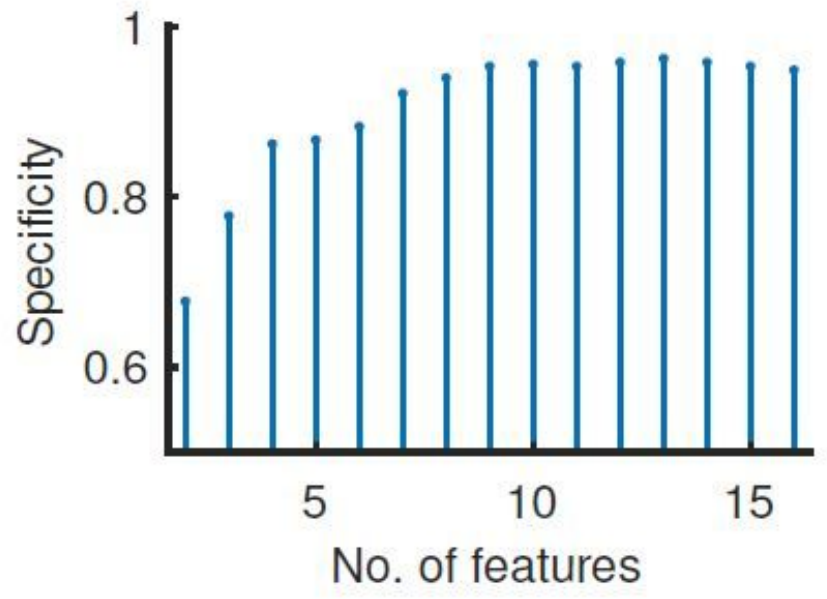

(c) Specificity

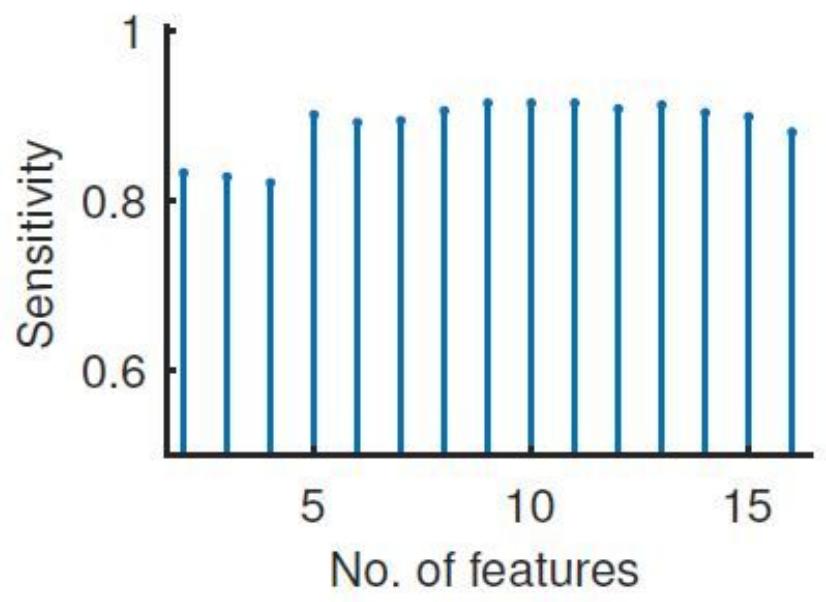

(b) Sensitivity

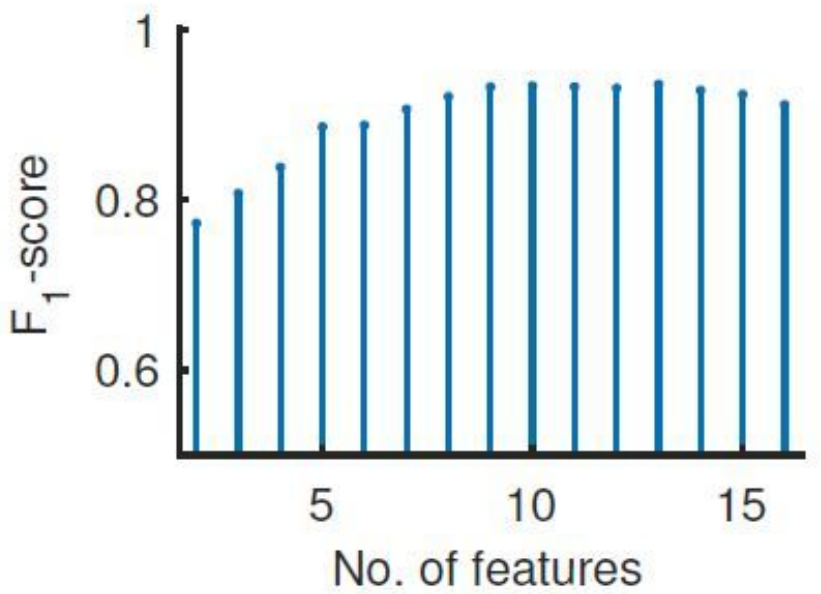

(d) $F_{1}$-score

Figure 1

Performance of the preterm-term birth classification obtained using the channel $\mathrm{s} 1$ of early period EHG data. 


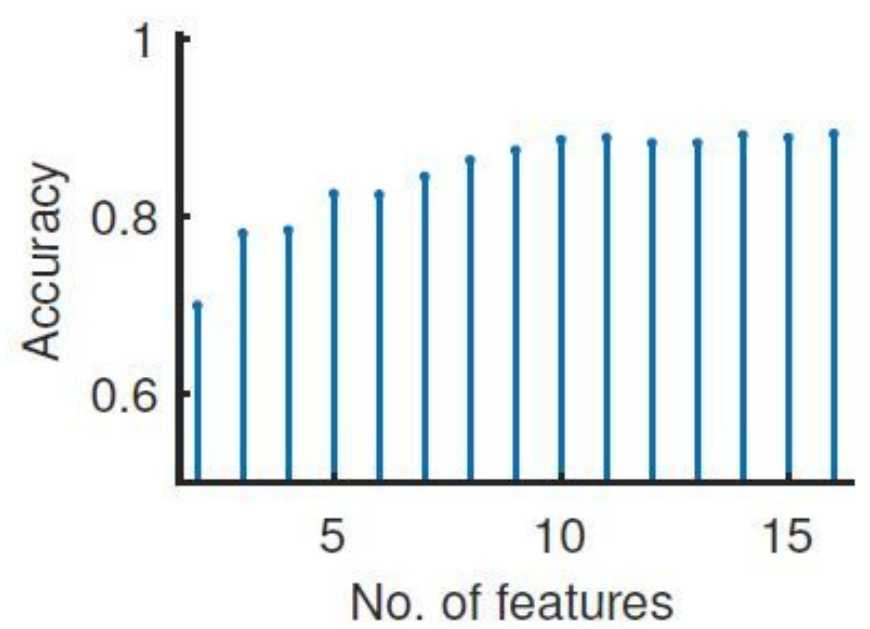

(a) Accuracy

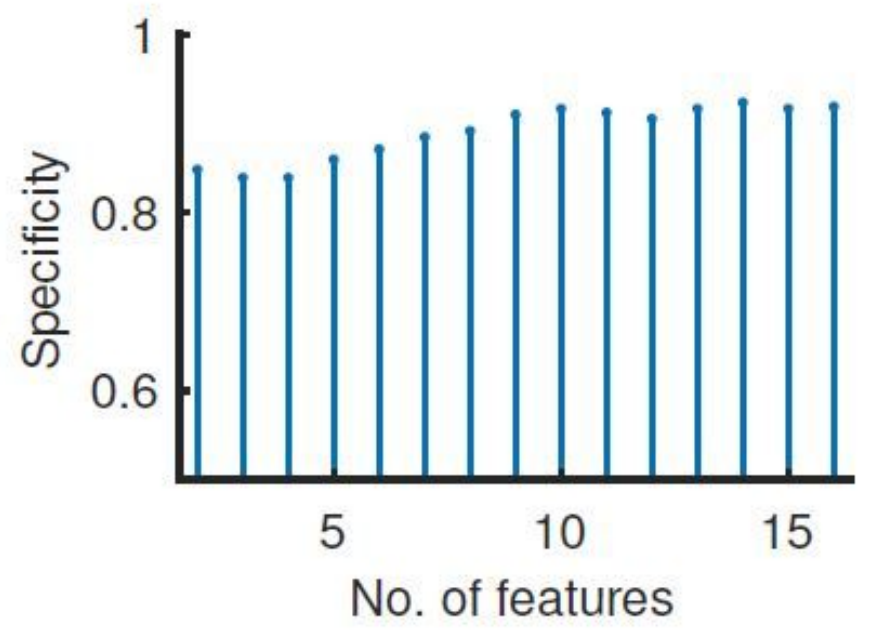

(c) Specificity

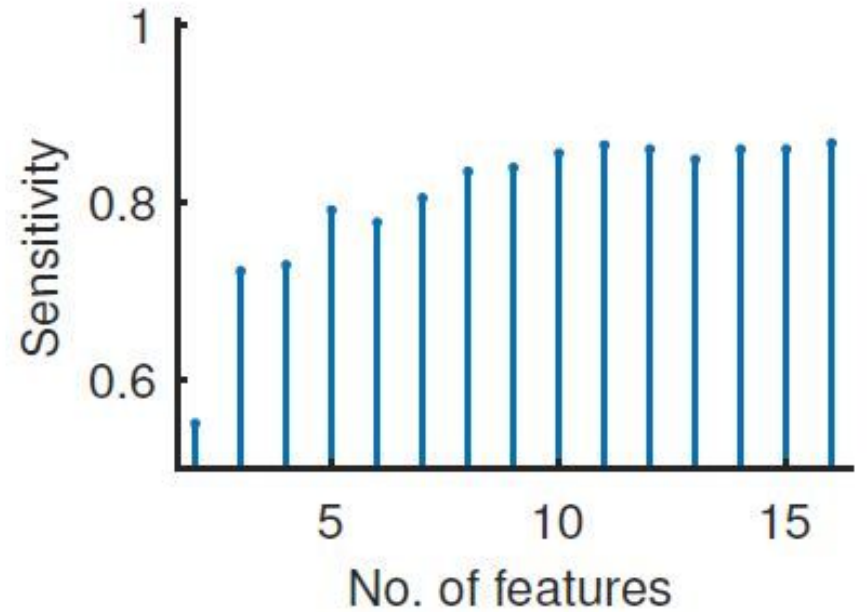

(b) Sensitivity

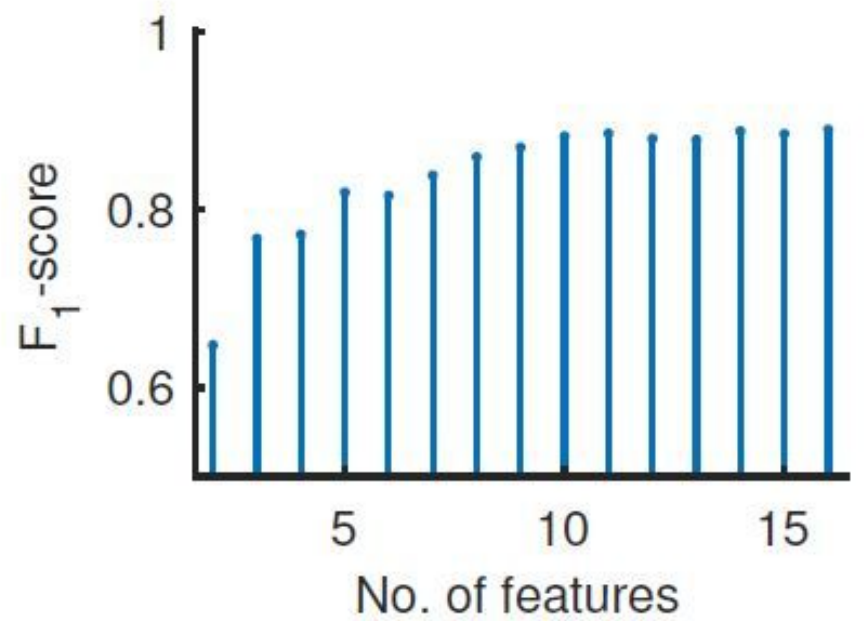

(d) $F_{1}$-score

Figure 2

Performance of the preterm-term birth classification obtained using the channel s2 of early period EHG data. 


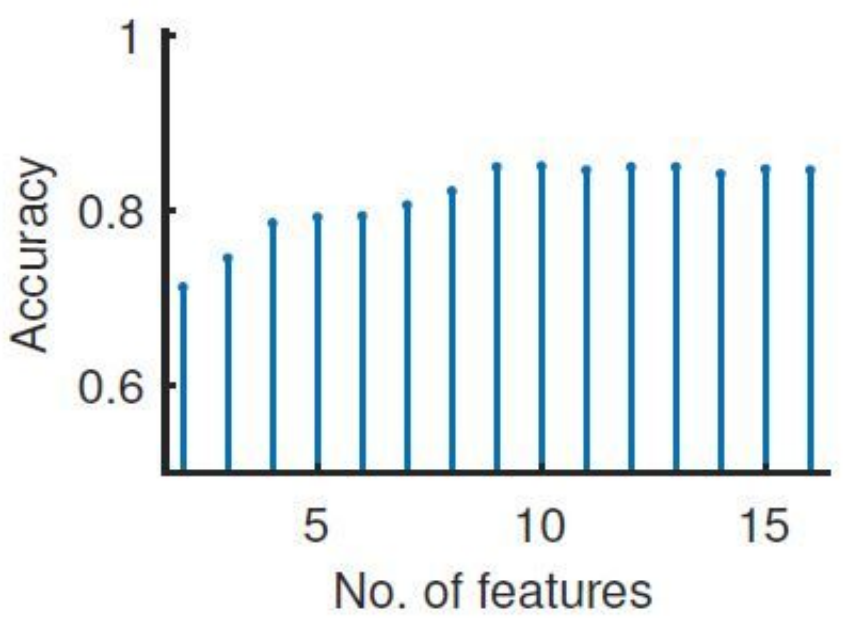

(a) Accuracy

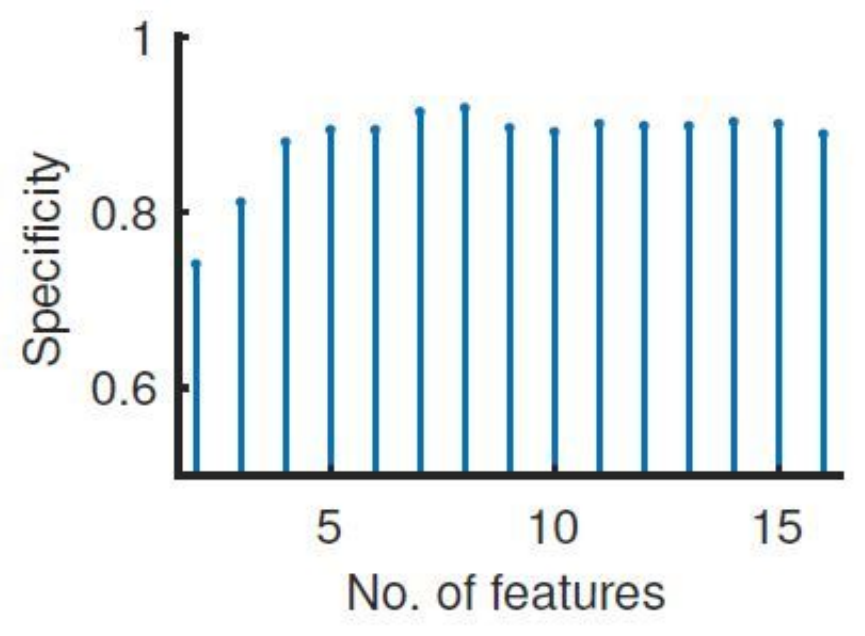

(c) Specificity

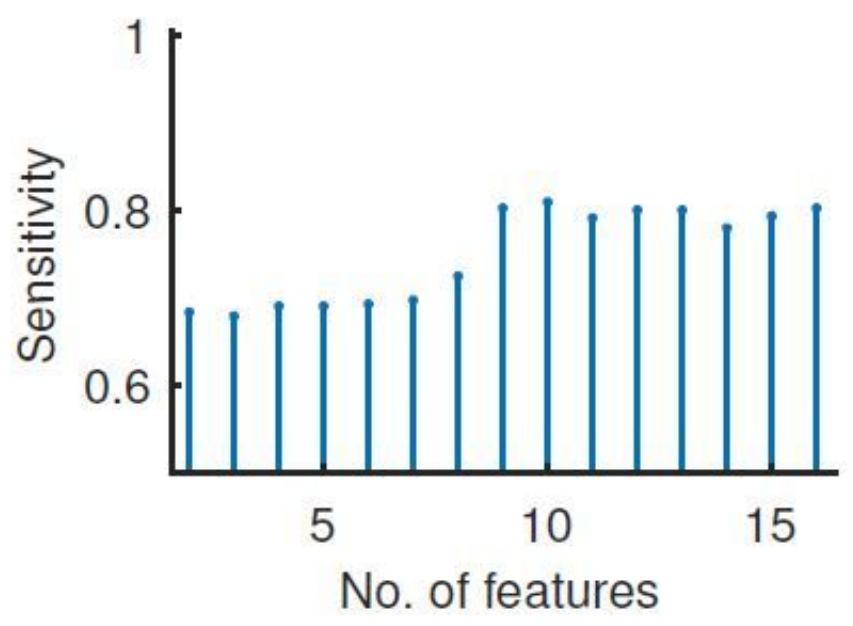

(b) Sensitivity

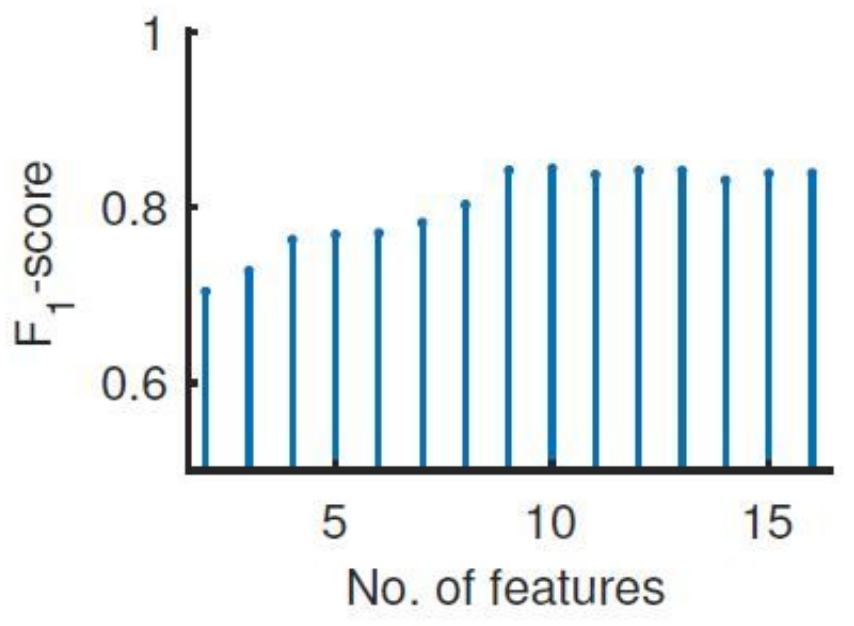

(d) $F_{1 \text {-score }}$

Figure 3

Performance of the preterm-term birth classification obtained using the channel s3 of early period EHG data. 


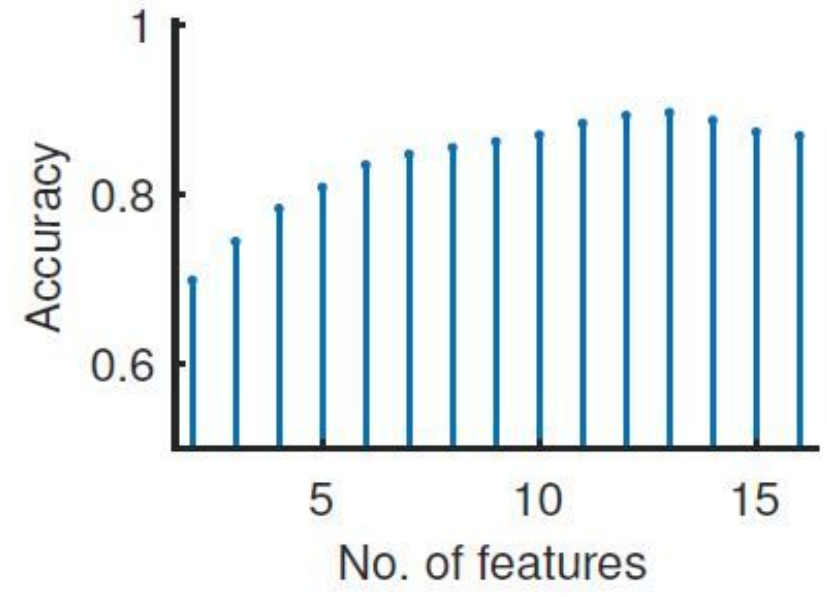

(a) Accuracy

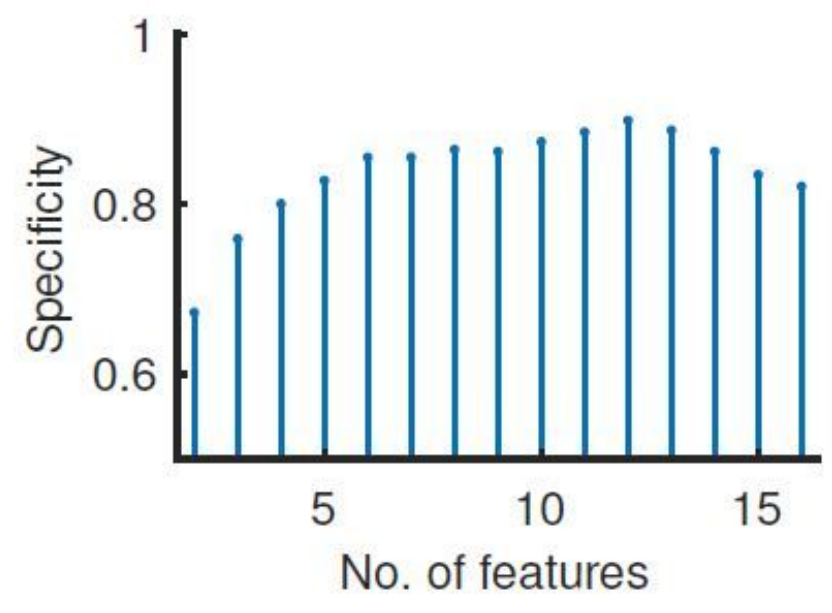

(c) Specificity

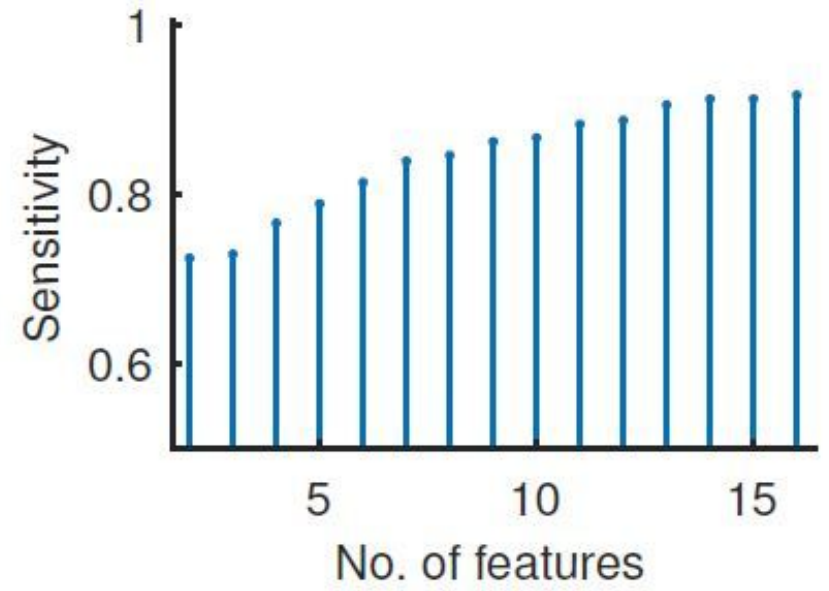

(b) Sensitivity

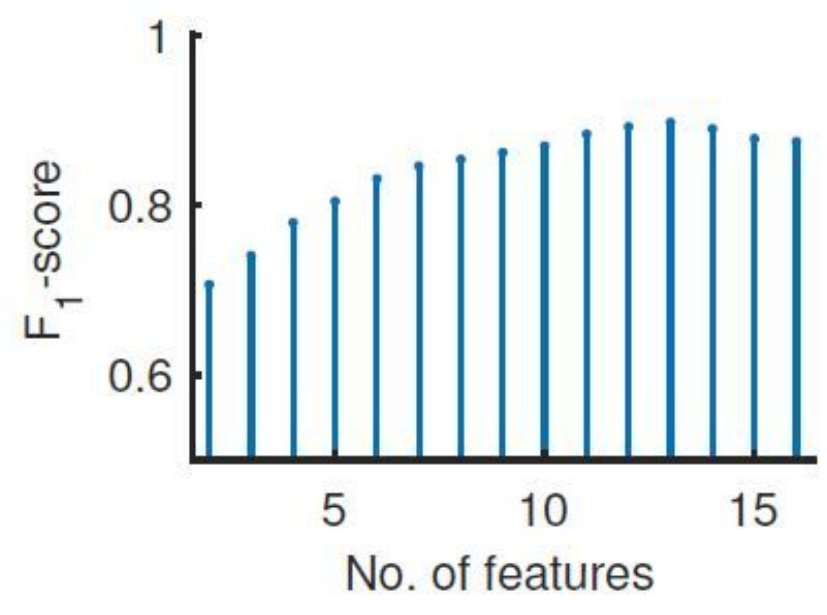

(d) $F_{1}$-score

Figure 4

Performance of the preterm-term birth classification obtained using the channel s1 of late period EHG data. 


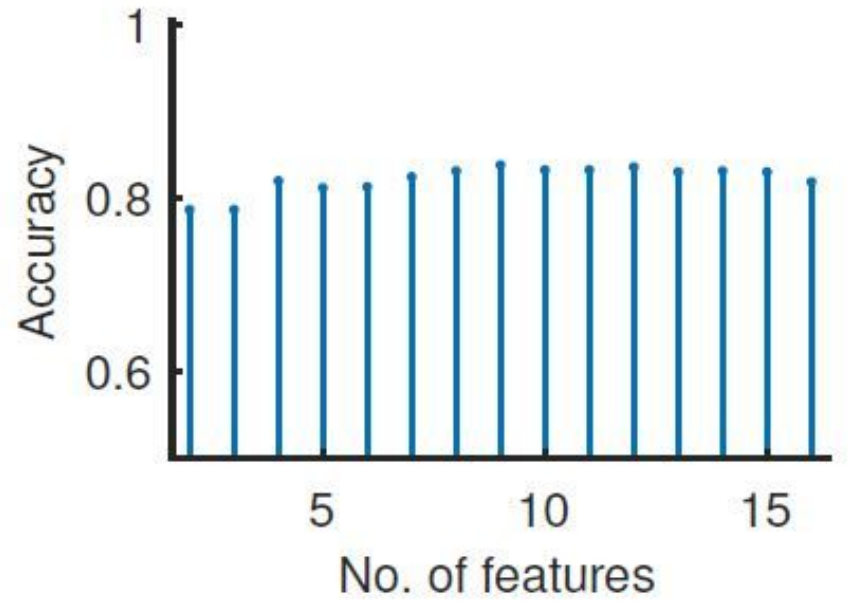

(a) Accuracy

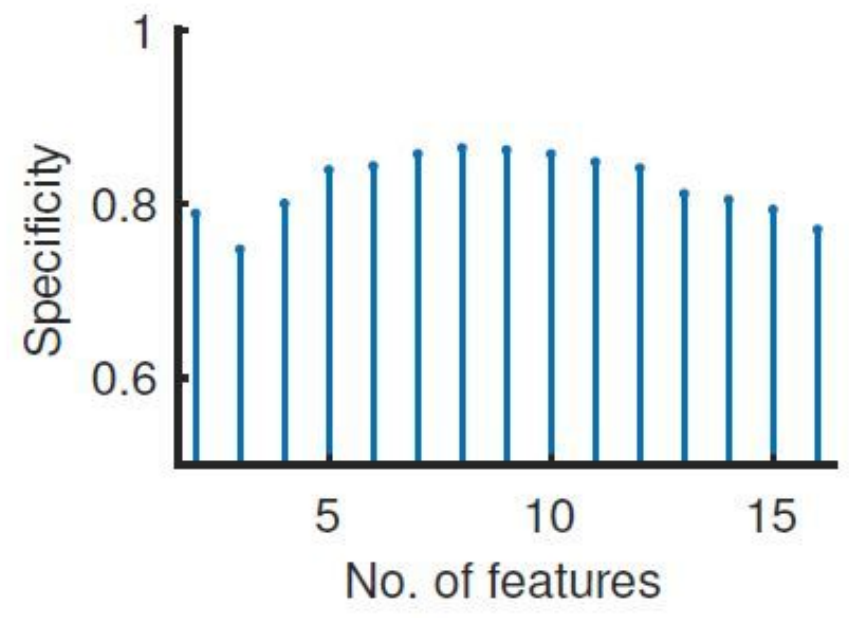

(c) Specificity

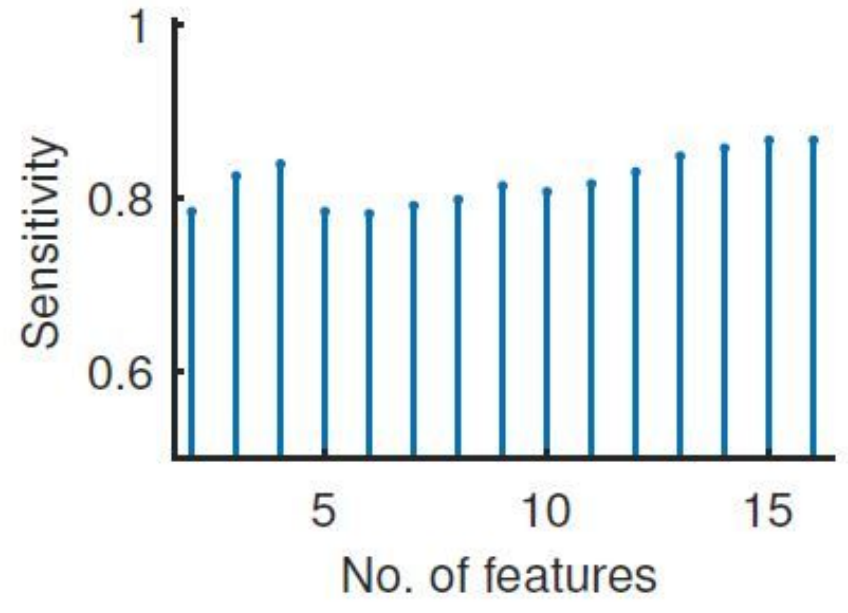

(b) Sensitivity

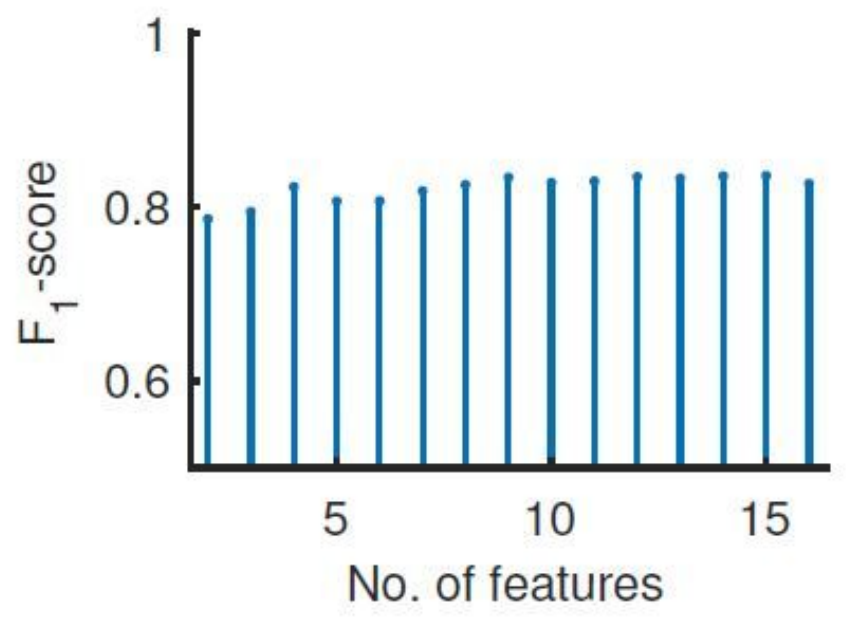

(d) $F_{1 \text {-score }}$

Figure 5

Performance of the preterm-term birth classification obtained using the channel s2 of late period EHG data. 


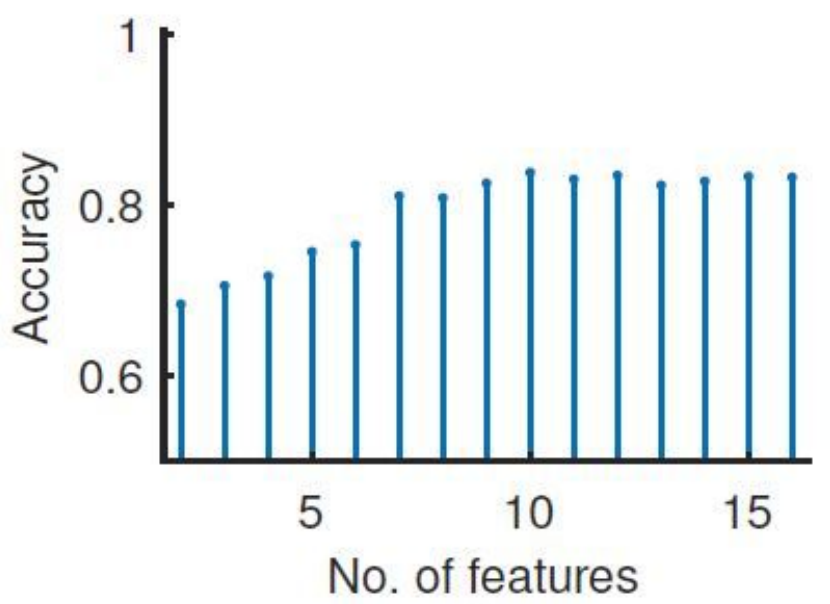

(a) Accuracy

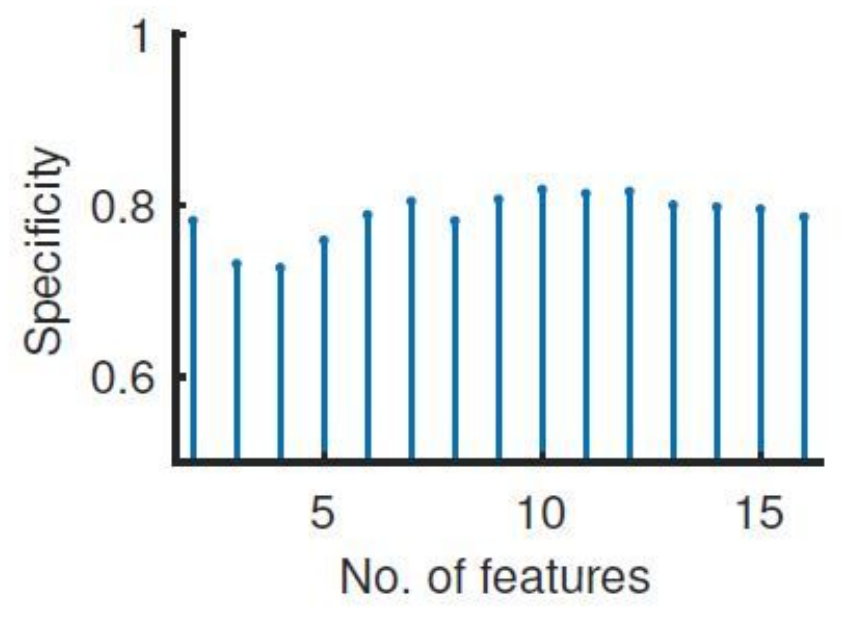

(c) Specificity

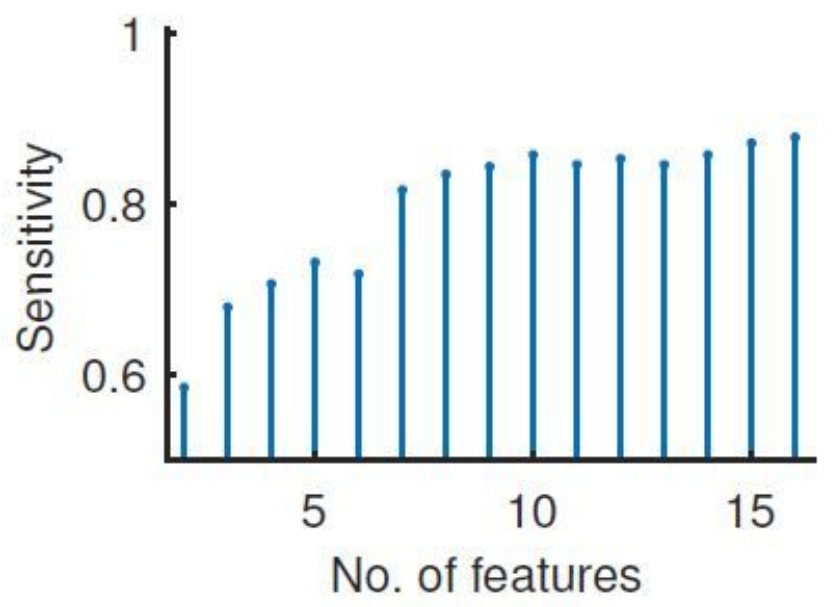

(b) Sensitivity

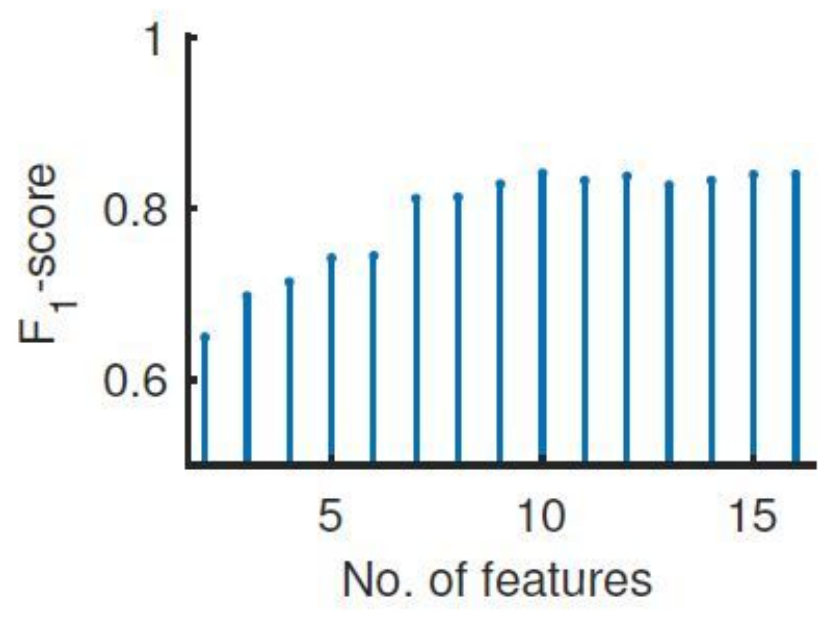

(d) $F_{1}$-score

Figure 6

Performance of the preterm-term birth classification obtained using the channel s3 of late period EHG data. 